\title{
Circuit
}

Musiques contemporaines

\section{Elektra 2002 : le poids du passé}

\section{Réjean Beaucage}

Volume 13, numéro 3, 2003

Électroacoustique : nouvelles utopies

URI : https://id.erudit.org/iderudit/902287ar

DOI : https://doi.org/10.7202/902287ar

Aller au sommaire du numéro

Éditeur(s)

Les Presses de l'Université de Montréal

ISSN

1183-1693 (imprimé)

1488-9692 (numérique)

Découvrir la revue

Citer cet article

Beaucage, R. (2003). Elektra 2002 : le poids du passé. Circuit, 13(3), 75-84.

https://doi.org/10.7202/902287ar

Ce document est protégé par la loi sur le droit d'auteur. L'utilisation des services d'Érudit (y compris la reproduction) est assujettie à sa politique d'utilisation que vous pouvez consulter en ligne.

https://apropos.erudit.org/fr/usagers/politique-dutilisation/
Cet article est diffusé et préservé par Érudit.

Érudit est un consortium interuniversitaire sans but lucratif composé de l'Université de Montréal, l'Université Laval et l'Université du Québec à Montréal. Il a pour mission la promotion et la valorisation de la recherche. https://www.erudit.org/fr/ 


\section{Elektra 2002 : le poids du passé Réjean Beaucage}

La quatrième édition du festival Elektra, une présentation conjointe de l'Association pour la création et la recherche électroacoustiques du Québec (ACREQ) et de I'Usine C, étalée sur six jours à Montréal s'est tenu entre les 7 et 16 novembre 2002. Se voulant un carrefour qui présente le dernier cri en matière d'arts électroniques et de culture numérique, Elektra nous a paradoxalement poussé, cette fois-ci plus que jamais, à coups d'hommages volontaires ou non à un passé pas si lointain, à nous poser une question troublante, à savoir : la "nouvelle musique électronique" plafonnerait-elle déjà? On en arrive en effet à se demander si les recours au passé ne sont pas, du moins dans plusieurs cas, des tentatives de se raccrocher à des œuvres qui reçurent en leur temps le sceau de l'originalité. Pour tenter de répondre à cette interrogation, nous examinerons donc ici les faits saillants de l'édition 2002 d'Elektra.

\section{Vidéomusique}

Le festival débutait le 7 novembre par une soirée vidéomusique dont la programmation présentait en majorité des créations. Ce nouveau courant qui se développe depuis les années 1990 met en relation l'image et le son, et nous présente ce que certains voient comme l'évolution la plus probable du médium électroacoustique. Bien sûr, plusieurs artisans de la musique électroacoustique prétendent depuis déjà longtemps faire du "cinéma pour l'oreille» (lire "sans image »|, mais justement, s'agissant de cinéma, pourquoi se priver ? D’autant plus que les équipements servant à créer et à transformer aussi bien l'image que le son sont aujourd'hui pratiquement les mêmes, ce qui explique que certains compositeurs commencent à créer leur propre univers visuel (et que beaucoup de vidéastes s'improvisent musiciens). Cependant, en sollicitant doublement le spectateur, on risque deux fois plus de se tromper, et on reconnaîtra avec les acousmaticiens que, pour être appréciées à leur juste valeur, certaines musiques requièrent toute l'attention de l'auditeur. De plus, la pauvreté d'une seule des composantes de l'œuvre suffit à en disqualifier l'ensemble. 
C'est pour cette dernière raison que Zack Settel a eu une mauvaise idée en recyclant sur écran triple The Secret Life of Objects, une œuvre visiblement conçue pour être projetée sur un seul écran. Les images conçues par François Roland et Joe Baar, dont on nous dit qu'ils sont très actifs dans la fabrication de publicités télévisées let ça se voit...।, ne gagnent rien au jeu de la multiplication et le compositeur n'arrive pas lui non plus, avec un enrobage discret, à les faire parler. Les œuvres suivantes, en utilisant trois projections synchronisées, donnaient véritablement la mesure de ce qu'il est possible d'attendre de ce médium. Les images de synthèse et la musique électronique quadraphonique de David Fafard, dans ZXOL, entraînent l'auditeurspectateur dans une rêverie abstraite, tandis que les images crues filmées par Thien Vu Dang (alias VJ Pillow) dans le métro de Tokyo pour SUBeat nous montrent sous tous ses angles la triste aliénation du métro-boulot-dodo réglé comme une horloge et dont la musique de Mathieu St-Arnaud appuie l'angoissant tic-tac. Les génériques étant montrés à la fin de chacune des œuvres et celles-ci n'étant pas projetées selon l'ordre du programme annoncé, il était évidemment très difficile de savoir chaque fois à qui l'on avait affaire. Dans mon calepin, après le début de Me and l, j'ai noté "Piché? ", puis, un peu plus loin, "très différent de paNi intiy $A^{\prime}$ ". C'était en effet assez différent de la manière habituelle de Jean Piché et pour cause, puisqu'il s'agissait en fait de l'œuvre d'un de ses étudiants à la maîtrise, Krystell Burlin, qui pourra certes comprendre cette méprise comme un compliment. Faisant déjà montre d'une grande virtuosité technique, il propose une œuvre complexe dans laquelle son et image sont en parfaite symbiose, se complétant à la perfection et se justifiant réciproquement.

Pour finir, après l'élève vint le maître. Je l'ai déjà dit ailleurs et je ne peux que le redire ici : il ne faut jamais rater la chance d'assister à la diffusion dans de bonnes conditions d'une œuvre de Jean Piché. Ce dernier a commencé à concevoir des œuvres intégrant la vidéo au début des années 1980, alors qu'il collaborait avec l'artiste américain Tom Sherman; durant les années 1990 il a choisi de prendre en charge également la confection de l'élément visuel de ses œuvres. Aujourd'hui, Jean Piché apparaît comme le chef de file de cette nouvelle pratique artistique.

Loin d'être un compositeur qui s'amuse en dilettante à concevoir une contrepartie visuelle pour sa musique, Piché fascine par la qualité graphique de ses productions. $C^{\prime}$ est au Jean-Paul Riopelle dernière manière que l'on pense devant certaines des images d'eXpress, véritable festival d'abstractions lyriques dansant aux sons des tablas et que viennent rythmer des images d'oiseaux "réels ". La tension monte dans un crescendo de guitare électrique (synthétique) qui mène à l'éclatement final. Et malgré le niveau élevé de perfection que Piché atteint dans eXpress, on monte encore d'un cran dans l'œuvre suivante, Bharat, dont le décor est planté en Inde, un coin du globe où l'artiste aime particulièrement puiser son inspiration. Ici, c'est la voix de nul autre que Mohandas Karamchand Gandhi qui sert de base à ce que I'on pourrait qualifier de documentaire surréaliste sur la vie au pays du Mahatma. Cette voix, enregistrée en avril 1947 alors qu'il donnait son dernier discours en langue
1. L'une des premières réalisations de Jean Piché, dont on a pu voir différentes versions à l'ACREQ entre 1995 et 1998. 
étrangère (évidemment, en anglais), revient de loin et donne au volet sonore de l'œuvre une texture bien particulière, et le violon d'Ivan Zavada contribue par ses lignes mélodiques à donner à l'ensemble une saveur indienne.

On sort d'une telle soirée avec l'impression que le médium n'en est plus à l'étape des essais et représente bien une voie d'avenir pour les créateurs bipolaires (image et musique) particulièrement dovés ou pour des équipes de collaborateurs spécialisés. Cependant, si l'équipement nécessaire à leur élaboration se simplifie et se multiplie de jour en jour, l'infrastructure nécessaire à la représentation de ce type d'œuvre risque fort de compromettre à long terme le développement de cette branche particulière de l'électroacoustique ou de limiter le nombre de créateurs à quelques happy few qui, non contents d'être marginalisés dans l'univers musical en général, seront aussi en minorité dans leur propre cercle.

\section{Ulysse}

La soirée du vendredi 8 novembre était entièrement consacrée au compositeur Christian Calon, qui présentait en diffusion acousmatique Time Well /La fontaine du temps/ et une œuvre mixte très ambitieuse Le projet Ulysse. On sait gré au directeur artistique de l'événement, Alain Thibault, de continuer à y inclure des œuvres en diffusion acousmatique, parce que la programmation très éclectique d'Elektra attire évidemment un auditoire très varié dont un bon pourcentage assiste probablement pour la première fois à ce type de concert durant lequel l'auditeur, complètement dépourvu de stimuli visuels, peut concentrer toute son attention sur le développement musical. Mais c'est surtout Le projet Ulysse qui promettait de retenir notre attention ce soir-là. Cette dernière fut cependant mise à rude épreuve par une œuvre plurielle qui attaquait le spectateur sur tous les fronts à la fois, incluant des voix et de la musique sur bande, mais aussi des acteurs et une chanteuse sur scène, et qui racontait en plusieurs langues une histoire inspirée de celle du Ulysse d'Homère, mais aussi de celui de James Joyce et incorporant même des références au satellite Ulysses de l'Agence spatiale européenne. L'œuvre contient aussi des références en forme $d^{\prime}$ hommages aux pionniers de la musique concrète. Beaucoup de matière. On regrette évidemment que l'on ait négligé de fournir au public un programme qui aurait pu offrir quelques pistes aux auditeurs qui n'avaient pas eu la chance de s'entretenir auparavant avec le compositeur sur ses intentions. De même, les écrans accrochés derrière la scène, mais restant désespérément blancs, auraient pu être utilisés pour aider le spectateur à se démêler. Ce dernier aurait sans doute mieux goûté le travail des comédiens Gabriel Gascon et Élisabeth Lenormand et celui de la chanteuse Shelley Hirsch, offrant une performance remarquable dans un répertoire assez éloigné de celui qu'elle fréquente habituellement. 
À ces trois voix s'exprimant en français et en anglais s'ajoutaient celles, sur bande, de Wajdi Mouawad, Fortner Anderson, Uli Aumüller (en allemand), Ned Bouhalassa, Chantal Dumas, Mario Gauthier et Geneviève Letarte. S'il était pratiquement impossible sans libretto d'apprécier pleinement le jeu des différentes couches de sens, la musique elle, construite à partir d'enregistrements du guitariste René Lussier, révélait un tout autre Christian Calon que celui auquel ses œuvres précédentes nous ont habitués, plus agressif et plus noir que jamais. Bref, une œuvre très dense, que l'on a hâte de réentendre sur disque, précisément à cause de sa trop grande complexité. Étant donné ses références à Joyce, on ne reprochera pas au compositeur d'en avoir «trop fait». II n'est pas dit qu'une œuvre doive être limpide dès sa première audition et ce genre de travail, où abondent les références, offre des plaisirs qui se laissent découvrir avec le temps. Le compositeur lui-même nous présentait une troisième version de ce projet sur lequel il travaille depuis 1995, et qui s'est déjà méritée le prix Lynch-Staunton du Conseil des Arts du Canada (1996) et le grand prix Marulic de l'Union européenne des radiodiffuseurs (1999). Peutêtre cette longue gestation aboutirattelle finalement à ce que cet Ulysse-à, comme ceux d'Homère et de Joyce, puisse, au bout de son périple, traverser le temps.

\section{Techno-pop}

Passons rapidement sur la première partie de la soirée du premier samedi d'Elektra, durant laquelle étaient projetés sur écran triple des vidéos conçus pour écran simple, de Chris Cunningham. Ce dernier a produit, depuis le milieu des années 1990, une douzaine de vidéos pour des artistes pops, des publicités télévisées et, plus récemment, un "vidéo d'art". Elektra présentait une courte rétrospective réunissant ses travaux avec Aphex Twin (Richard D. James) et la chanteuse Biörk. Le style narratif des vidéos de Cunningham est peu fréquent sur les ondes de stations de télévision comme MTV aux États-Unis ou Musique Plus chez nous, et c'est peutêtre pour cette raison que le public habituel de ces stations les trouvent si originaux, mais, outre quelques effets spéciaux tout à fait spectaculaires (comme dans All is Full of Love, de Björk) le médium est certes mieux servi par les artistes qui étaient présents à la soirée d'ouverture du festival.

La programmation de la soirée du samedi, avec des noms bien connus des jeunes qui fréquentent les discothèques à la mode, est conçue avec l'objectif avoué ${ }^{2}$ d'en faire la soirée qui rapportera au festival le meilleur pourcentage de ses recettes. On ne peut que déplorer que le manque d'autonomie financière d'un événement de l'ampleur d'Elektra oblige son directeur artistique à rogner sur la qualité pour assurer un rendement qui satisfasse ses bailleurs de fonds. Mais on peut difficilement reporter sur Alain Thibault la responsabilité de cette situation.

2. Extrait d'une entrevue accordée à Alain Brunet et publiée dans la Presse du jeudi 7 novembre 2002, dans le cahier « Arts et spectacles", p. C6, sous le titre "Elektra: angle aigu, large vision »: « Nous présentons bien sûr des trucs plus commerciaux comme le funk déjanté du collectif anglais 
Quoi qu'il en soit, c'est à la formation britannique Super_Collider, qui présente des chansons pops aux rythmes électro-funk, que l'on avait confié ce mandat rassembleur. De quoi donc s'agitil ? Un bassiste (James Stephenson) et un batteur (Grant Allardyce) accompagnent ici le duo formé de Cristian Vogel (claviers, tournedisque) et Jamie Lidell (voix). Pablo Fiasco se charge de retransmettre en direct des images du concert et des images préenregistrées sur l'écran triple placé derrière la scène. Mais attendez... Basse, batterie, claviers, un chanteur qui s'amuse à porter différents déguisements, trois écrans en haut de la scène... Auraiton utilisé une machine à voyager dans le temps pour me renvoyer en 1974 assister au concert The Lamb Lies Down on Broadway de Genesis? On parle d'un concert qui se donnait il y a près de 30 ans... Celuilà était apparemment assez en avance sur son époque pour qu'une formation montréalaise reprenne intégralement le même programme en 2001 et entreprenne de le présenter jusqu'en Europe ${ }^{3}$. II faut dire qu'à défaut des moyens technologiques disponibles aujourd'hui, les concepteurs du spectacle de Genesis avaient de l'imagination, et arrivaient à faire avec des diapositives quelque chose de beaucoup plus intéressant que $M$. Fiasco avec son attirail vidéo. Bien sûr, il y a 30 ans, le gros du public d'Elektra n'allait pas beaucoup au concert, fût-il de rock progressif; aussi, ce genre de spectacle peut-il se donner des airs de nouveauté. Je doute fort que l'histoire retienne ne serait-ce qu'une trace de Super_Collider...
Super_Collider ou la programmation de notre party de clôture (le samedi 16 novembre), mais le reste est beaucoup plus expérimental. Ça nous prend évidemment un ou deux programmes qui nous permettent de vendre plus de billets et plus de bière. Ceux qui nous subventionnent veulent qu'on génère des revenus autonomes et il n'y a pas 56 receltes pour y arriver ", fait observer le directeur artistique.

[...]

«Avec le modeste budget dont nous disposons (250 $000 \$$ ), précise-til en outre, nous essayons de présenter ce que nous considérons de plus intéressant. Le côté visuel, à ce titre, a pris une grande place à Elektra, bien que la musique y demeure centrale. En fait, nous cherchons des œuvres et des performances où d'autres médiums que la musique sont intégrés. "

3. La formation The Musical Box a repris, en 1991, les concerts que donnait le groupe rock britannique Genesis au début des

\section{Automates et non-humain}

Le second week-end d'Elektra nous a permis de faire l'étrange rencontre des deux "Juke Bots" du collectif Robot Lab (Matthias Gommel, Martina Haitz et Jan Zappe). Bien que l'un des membres du trio, Matthias, ait étudié la musique, il ne s'agit pas à proprement parler d'une performance musicale, mais plutôt d'une expérience sociologique. En effet, le but de Robot Lab est de créer des rencontres entre l'humain et la machine, afin d'étudier le comportement du premier dans un contexte où c'est cette dernière qui fait acte de "création", tandis que l'humain se contente d'être spectateur. Les machines utilisées sont de véritables robots industriels (fabriqués par la firme allemande KUKA) que les membres du trio détournent de leurs fonctions habituelles, mais en prenant soin de conserver intactes leurs capacités à effectuer des travaux d'une précision extrême. Ici, donc, les bras pneumatiques sont programmés pour saisir des disques de vinyle (chacun dispose d'un "choix" de 11 disques judicieusement placés autour de luil et les faire jouer sur un tourne-disque; ils peuvent accélérer et ralentir la vitesse de rotation du disque dans un sens ou dans l'autre, mais tous ces gestes sont programmés à l'avance selon une séquence définie. Les robots ne sont pas dotés, par exemple, de programmes aléatoires qui rendraient leur jeu imprévisible. II serait possible de les perfectionner en ce sens, mais, comme années 1970 avec le chanteur Peter Gabriel. TMB apporte un soin maniaque à ses interprétations, respectant intégralement la mise en scène des concerts de Genesis, comme s'il s'agissait de comédies musicales. En 2002, TMB se produisait avec beaucoup de succès en Angleterre et prévoit une tournée en Allemagne en 2003. 
je le disais plus tôt, là n'est pas le propos des membres du collectif. Si le travail en général de Robot lab peut être compris comme une critique de la condition humaine, il ne s'agit pas, ici en particulier, d'une critique dirigée à l'endroit du monde des DJ, comme on a pu le lire ailleurs ${ }^{4}$. Robot lab a conçu avec les mêmes machines des installations bien différentes, où, par exemple, un robot jouant les peintres de foire fait le portrait (très réaliste) des participants qui se placent devant lui (comble de l'ironie, chaque portrait est détruit dès qu'il a été complété). Le spectateur ou le participant est invité à rélléchir sur le concept de "création "; une machine peutelle être pourvue d'une imagination créatrice? Nous n'en sommes apparemment pas encore là et les "Juke Bots " ne sont précisément pas équipés des fonctions qui pourraient permettre d'en donner véritablement l'illusion. C'est d'ailleurs un reproche que plusieurs spectateurs ont formulé après avoir assisté à la performance des robots, il est dommage que tout cela n'aille pas un peu plus loin, que tout cet attirail en reste à la démonstration de prototype d'exposition scientifique.

La soirée allait se poursuivre avec un véritable DJ "humain", A-Trak (Alain Macklovitch), qui allait prouver sans équivoque que l'équipe de Robot lab en aura pour des années encore à perfectionner ses machines si son but est de créer des cyber-DJ capables de surpasser le "facteur humain ". Parce que si les «Juke Bots" sont encore loin d'être aussi redoutables qu'un Big Blue dans une partie d'échecs, A-Trak est au moins un Garry Kasparov du tourne-disque ! Récipiendaire de plusieurs titres de "champion du monde", dont le premier fut remporté en 1997 alors qu'il n'avait que 15 ans, A-Trak devait en principe participer à une improvisation en duo avec Naut Humon ${ }^{5}$ en début du programme préparé par ce dernier, mais Humon a sans doute préféré le laisser s'exprimer en solo puisque, de toute façon, le DJ ne semblait pas d'humeur à laisser beaucoup de place ${ }^{6}$. À l'aide de deux platines, d'un mélangeur et d'un échantillonneur contrôlé par des déclencheurs qu'il manipulait avec le pied, A-Trak a donné une formidable démonstration non seulement de son savoir-faire, mais aussi des incroyables possibilités de ses instruments. Les images de chacune de ses platines et de son mélangeur étant reproduites sur les trois écrans géants, le public pouvait observer son travail dans les moindres détails et ceux qui ne le connaissaient pas, ou qui ne s'étaient jamais intéressés à l'art particulier des DJ, ont immédiatement compris qu'ils avaient affaire à un grand virtuose. Le DJ, comme les "Juke Bots", donnait une démonstration; il s'agissait très probablement de la même routine qu'il sert dans les compétitions de DJ qu'il est habitué de remporter et ce fut de sa part un choix judicieux. Comme un gymnaste aux Jeux olympiques qui doit, lors de sa prestation, faire une série de figures imposées et, idéalement, faire preuve d'originalité, le DJ a exécuté toute la gamme des mouvements possibles. Paradoxalement, le résultat musical était assez frustrant pour les véritables amateurs de ce genre de travail, puisque chacune des différentes sections de sa démonstration était très courte, mais les néophytes découvraient là un univers auquel même les écrits de Pierre Schaeffer ne les avaient pas préparés!

Cettle soirée était placée sous le signe de la " reconstruction ${ }^{7}$ », un thème cher à Naut Humon, qui en avait conçu le programme. Après le détournement surréaliste
4. Dans Le Devoir du 18 novembre /cahier "Culture», p. B8), sous le titre "Arts technologiques - Comme un poème de Rilke ou d'Éluard ", François Tousignant écrivait: "Au début de la soirée, deux robots nous ont décortiqué l'« art " des DJ. Quelle satire de ce genre. II a probablement fallu un bon culot au vrai DJ, A-Trak, pour venir faire sa performance ensuite. Cela montre toute la vanité et l'incroyable fatuité de ce mode de faire de la musique consommable, en exemplifiant le conformisme et le mécanisme, le non-besoin d'imagination et la stérilité à laquelle cette superficialité est dorénavant forcément vouée. "

5. Fondateur de l'étiquette de disques Asphodel et éminence grise des nouveaux courants d'art médiatique.

6. On a compris lorsqu'il est revenu pour la soirée de fermeture, et qu'il a redonné exactement la même prestation qu'il nous avait donné chaque fois, une démonstration maintes fois répétées. Cela n'enlève absolument rien à son très grand talent, d'autant plus que l'on pourrait y voir un geste ironique de sa part, puisque les « Juke Bots » aussi ont répété la même routine lors de leurs trois prestations...

7. Naut Humon utilise, en anglais, le terme reconstructive. 
des robots industriels de la compagnie KUKA et la création en direct par A-Trak d'une pièce musicale à partir de bribes d'œuvres enregistrées sur disque, Humon allait nous livrer sa relecture du Persepolis de lannis Xenakis. L'étiquette américaine Asphodel, que dirige Naut Humon, a fait paraître un premier volume de remixages de la pièce Persepolis ${ }^{8}$ et un deuxième est en préparation. L'idée de base de sa performance consistait donc à " recomposer » Persepolis ou, plus précisément, à rendre hommage à l'esprit de l'œuvre et à celui de son compositeur. Utilisant comme matériau de base l'œuvre elle-même, les remixages qui en ont été inspirés (tant du premier volume que du second) et quelques ingrédients de son propre cru, le tout manipulé en direct, Naut Humon a diffusé dans une pénombre semi-acousmatique une improvisation où l'on sentait en effet le geste et l'intention de Xenakis. Voilà bien une avenue peu explorée en matière de musique acousmatique, que celle de l'improvisation à partir de plusieurs sources. Cette seule innovation était en soi rafraîchissante, et le résultat sonore ("musical " semble déplacé ici), étant donné le matériau de base et l'extraordinaire puissance du système d'amplification d'Elektra (dont Naut Humon semblait décidé à tester l'efficacité) fut proprement hallucinant. Elektra se fait un point d'honneur de présenter à chaque édition un concert durant lequel le son agresse l'auditeur jusqu'au seuil de la tolérance. Ce n'est pas, on aime à le croire, par pur sadisme, mais bien parce que l'expérience du concert prend vraiment dans ces circonstances un tout autre sens. Ressentant les basses jusque dans la moelle de ses os, l'auditeur vit une expérience psycho-acoustique qui ne peut être induite autrement; on a l'impression d'être touché physiquement par la "musique»; le son, c'est certain, n'a jamais été aussi concret.

Naut Humon reprenait ensuite le même exercice en le transposant à l'œuvre vidéomusique NoiseGate, du duo autrichien Granular Synthesis (Kurt Hentschläger et Ulf Langheinrich). NoiseGate a été conçu comme une installation interactive, les mouvements des spectateurs étant utilisés pour exercer des variations tant sur les images que sur la musique qui les accompagne. L'œuvre originale montrait, sur un nombre d'écrans variable, une tête d'homme qui semblait emprisonnée par le cadre de l'image et qui chercherait à en sortir. La musique était un long accord tenu secoué d'imperceptibles variations (un drone) et le résultat était lourd et angoissant. Naut Humon en a fait tout autre chose, par accélération, modifications diverses et ajouts tant à l'image qu'au son. Le mot "déferlement" semble faible pour rendre compte de la tornade visuelle et sonore qui résulte de ses manipulations. Ici, c'est aussi le sens même de l'œuvre qui est détourné. II ne s'agit plus d'un hommage, mais d'une appropriation.

À la fin de cette soirée, on réalise que les trois prestations, des «Juke Bots » à Naut Humon en passant par A-Trak, ont utilisé comme matière première des œuvres conçues par d'autres artistes dans un passé plus ou mois récent: L'utilisation des disques chez A-Trak n'est pas discutable, puisque c'est elle qui engendre la pratique; idem pour les robots, qui en sont la caricature. Chez Naut Humon, I'utilisation d'une œuvre antérieure comme matière première est assimilable à ce qui se fait chez beaucoup de compositeurs postmodernes de musique instrumentale. Mais le
8. Voir la recension page 85 
postmodernisme ou le bruitisme (le remixage de NoiseGate pouvant être considéré comme du bruitisme visuel) ne sont plus vraiment des nouveautés, et l'œuvre ne doit ici son caractère novateur qu'à des progrès techniques qui touchent la reproduction, progrès qui permettent de décortiquer et de singulariser les différentes qualités de bruit. C'est évidemment dans la forme que tout se joue. lci, le volume sonore ajoute une nouvelle dimension, mais on ne pourra pas longtemps, année après année, se contenter d'ajouter des décibels.

\section{Électro-humain}

Décidément, Elektra ressemble de plus en plus à un festival de cinéma. Le vendredi 15 novembre, ce sont quatre écrans qui nous attendaient dans la grande salle de I'Usine C. Le trio Battery Operated (Toby Heys, Bérengère Marin Dubuard et Thomas Couzinier) y présentait d'abord Hydraulic Melancholy, une création dont le meilleur aspect était certainement le volet visuel, mais il a déjà été beaucoup trop question de vidéo dans cet article. Parlons plutôt de théâtre. Parce que c'est presque à une performance théâtrale que nous conviaient les Américains Joshua Kit Clayton Imusicien et programmeur informatique) et Sue Costabile (photographe et artiste vidéo) avec leur performance intitulée Interruption (2002). La photographe a expliqué en entrevue la genèse de l'œuvre par un désir de ramener la présence humaine à l'avant-plan et de travailler le son et l'image simultanément, plutôt que l'un après l'autre comme c'est souvent le cas ${ }^{9}$. Les deux artistes sont donc sur scène, couchés sur des tables avec un micro suspendu près du visage et une caméra vidéo qui filme celvi-ci en gros plan. Ce sont leurs voix qui contrôlent (ou interrompent), au moyen d'une interface conçue par Clayton, le déroulement sonore et celui des images projetées sur les écrans, soit leur visage en direct ou un enregistrement vidéo. Les images préenregistrées sont parfaitement banales, le but étant d'explorer l'étrangeté de la "vie de tous les jours". Le résultat est en effet pour le moins étrange, la voix des artistes se mêlant à un environnement sonore très abstrait ef la présence des artistes, les gros plans de leurs visages, installant un climat d'intimité factice. Encore une fois, c'est une expérience émotionnelle que l'on propose, dans laquelle le son n'est qu'une composante parmi d'autres.

C'est une autre prestation plus proche de l'art de la performance qui terminait cette soirée. L'artiste sonore canadien Gordon Monahan, qui a beaucoup travaillé sur les qualités physiques du son, présentait Speaker Swinging, une autre œuvre ayant un rapport évident avec le passé, puisqu'elle a été créée en 1982. Une œuvre très simple dans laquelle trois participants font tournoyer autour de leur tête un haut-parleur au bout de son câble d'alimentation. Le compositeur diffuse avec un générateur d'ondes un son pur dans chaque haut-parleur qui, en tournant, produit

9. Dans une entrevue accordée à Raf
Katigbak et publiée dans l'édition du
14 novembre 2002 du Montreal Mirror
(vol. 18, n' 23): "Our main motivation
was two-fold. One was to do something that
was more of a theatre piece and more of a
human performance element to it, and the
other was to do something that merged
audio and video from the beginning, ins-
tead of trying to link them together later,
which is often the case. Also, the tools were
available, so it seemed like an interesting
way to try and use them. "

9. Dans une entrevue accordée à Raf Kaligbak et publiee dans lédition du 14 novembre 2002 du Montreal Mirror (vol. 18, n²3) : «Our main motivation was two-fold. One was to do something that was more of a theatre piece and more of a human performance element to it, and the other was to do something that merged audio and video from the beginning, instead of trying to link them together later, available, so it seemed like an interesting way to try and use them." 
un effet Doppler. Chaque hautparleur est aussi muni d'une lampe qui, dans l'obscurité, permet de suivre la "trajectoire " du son. Il y a ici un élément de risque dans la manœuvre qu'opèrent les participants et qui croît avec la durée de la performance. Leur endurance étant mise à rude épreuve, le spectateur a l'impression d'observer des esclaves se livrant à quelque incompréhensible tâche. Une œuvre très simple en effet, mais diablement efficace et qui a connu un très grand succès ce soir-là. J'ai pu vérifier que plusieurs spectateurs étaient très surpris d'apprendre que l'œuvre avait été créée 20 ans plus tôt...

\section{Coda}

Elektra, dont la devise était, lors de la deuxième édition du festival en 2000, The Future is Beautiful, ne nous a pourtant pas donné cette fois-ci une image très enthousiasmante de l'avenir. D'abord en faisant de trop fréquents détours par le passé, ce qui donne l'impression d'être arrivé à une limite esthétique infranchissable, mais aussi en se complaisant dans un axe paradigmatique où le sentiment de claustration (Granular Synthesis, Clayton/Costabile) ef l'esclavagisme (Monahan) se conjuguent avec la déshumanisation ("Juke Bots", Naut Humon). En commençant la dernière soirée avec la projection du long-métrage Alphaville (1965) de Jean-Luc Godard, paresseusement "remixé » par Scanner (Robin Rimbaud, qui n'ajoutait que quelques éléments à la bande son du film), le directeur artistique poursuivait dans la même voie. On avait en effet l'impression de revenir en arrière et d'avoir la même vision traumatisante de l'«avenir" que celle que l'on trouve dans certaines œuvvres de science-fiction, du Big Brother de George Orwell ${ }^{10}$ au Terminator de James Cameron "'. Et peut-être aussi l'impression d'assister en direct à la fin d'une certaine avant-garde.

10. Dans Nineteen Eighty-four (1948).

11. Film réalisé en 1984. Fortement inspiré de plusieurs œuvres de l'auteur américain Harlan Ellison. 


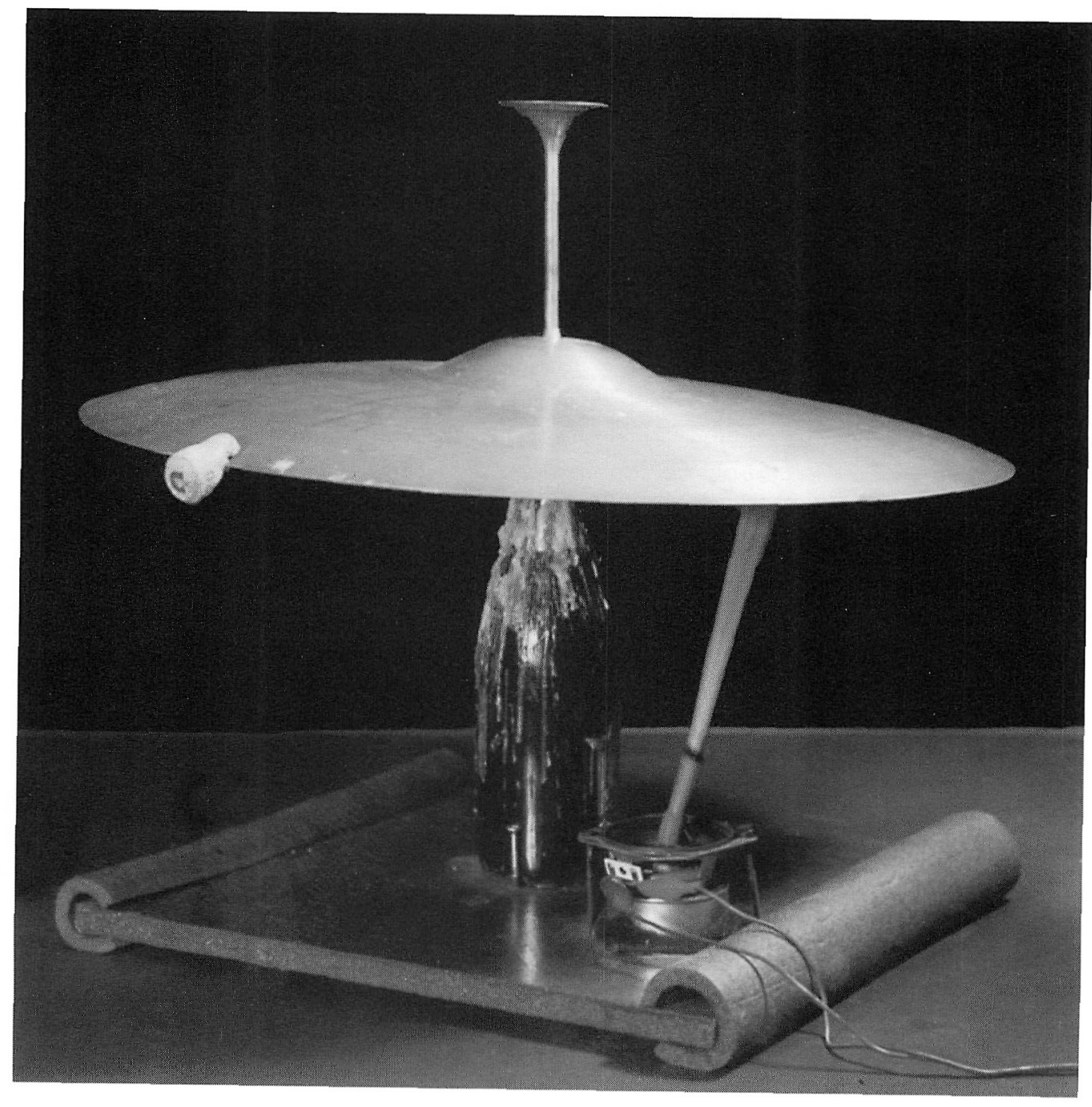

